\title{
Qualidade da Atenção Primária à Saúde infantil em estados da região Nordeste
}

\author{
Quality of primary care for child health \\ in states in the Northeast region
}

Fabiane do Amaral Gubert (https://orcid.org/0000-0003-3016-9619) ${ }^{1}$

Valter Cordeiro Barbosa Filho (https://orcid.org/0000-0002-4769-4068) ${ }^{2}$

Rejane Christine de Sousa Queiroz (https://orcid.org/0000-0003-4019-2011) ${ }^{3}$

Mariana Cavalcante Martins (http://orcid.org/0000-0001-8234-8980) ${ }^{1}$

Renata de Sousa Alves (https://orcid.org/0000-0003-0630-1499) ${ }^{1}$

Isaura Letícia Tavares Palmeira Rolim (https://orcid.org/0000-0002-8453-2543) ${ }^{3}$

Maria do Socorro Vieira Lopes (https://orcid.org/0000-0003-1335-5487) ${ }^{4}$

Anya Pimentel Gomes Fernandes Vieira-Meyer (https://orcid.org/0000-0003-4237-8995) ${ }^{5}$

\footnotetext{
${ }^{1}$ Universidade Federal do Ceará. R. Alexandre Baraúna 1115, Rodolfo Teófilo. 60430-160 Fortaleza CE Brasil. fabianegubert@hotmail.com ${ }^{2}$ Instituto Federal de Educação, Ciência e Tecnologia do Ceará. Fortaleza CE Brasil. ${ }^{3}$ Universidade Federal do Maranhão. São Luís MA Brasil.

${ }^{4}$ Universidade Regional do Cariri. Crato CE Brasil.

${ }^{5}$ Fundação Oswaldo Cruz Ceará. Eusébio CE Brasil.
}

\begin{abstract}
The scope of this article is to compare the assessment of users of health services who participated in the PMAQ-AB on child health in the states of the Brazilian Northeast. It is a cross-sectional study conducted with secondary data based on interviews with 5,116 users of 4,190 Family Health Teams in the nine states of the Northeast macroregion. Fourteen questions on the medical monitoring of children from 0 to 2 years old were evaluated. Proportions and 95\% confidence intervals were calculated for each Unit of the Federation of the Brazilian Northeast. The indicators with the highest proportions were the Guthrie PKU test performed within seven days of life (84.4\%), children always accompanied by the same professionals (79.1\%), monitoring expected growth and development for age (84.9\%), dietary guidance $(86.3 \%)$ and up-to-date vaccination (95.3\%). The indicators with the lowest proportions were guidance on the best position for the child to sleep in (45.7\%) and whether or not the child's health handbook (46.9\%) was received. In all indicators, there were significant differences between the states analyzed. There are differences in the quality of child health care on offer according to the evaluation of users participating in the $P M A Q-A B$ between states in the Northeast.

Key words Primary Health Care, Health assessment, Child health, Pediatric nursing, Health services
\end{abstract}

Resumo $O$ objetivo deste artigo é comparar a avaliação das usuárias que participaram do PMAQ-AB acerca da saúde infantil entre os estados da macrorregião Nordeste do Brasil. Estudo transversal, realizado com dados secundários, a partir de entrevista com 5.116 usuárias de 4.190 Equipes de Saúde da Família que atuam nos nove estados da macrorregião nordeste. Quatorze perguntas acerca do acompanhamento da criança de 0 a 2 anos de idade foram avaliadas. Foram calculados proporções e intervalos de confiança de $95 \%$ segundo Unidade da Federação da região Nordeste do Brasil. Os indicadores com maiores proporçôes foram teste do pezinho realizado em até sete dias de vida (84,4\%), criança sempre consultada pelos mesmos profissionais (79,1\%), acompanhamento do crescimento e desenvolvimento esperado para a idade (84,9\%), orientação alimentar (86,3\%), vacinação em dia (95,3\%). Os indicadores com as menores proporções foram orientação sobre a melhor posição para a criança dormir $(45,7 \%)$ e se recebeu a caderneta de saúde da criança (46,9\%). Em todos os indicadores, houve diferenças significativas entre os estados analisados. Existem divergências na qualidade do cuidado ofertado à saúde infantil segundo a avaliação das usuárias participantes do PMAQ-AB entre estados do Nordeste.

Palavras-chave Atenção Primária à Saúde, Avaliação em Saúde, Saúde da Criança, Enfermagem Pediátrica, Serviços de Saúde 


\section{Introdução}

A avaliação do cuidado à saúde da criança possibilita qualificar o processo de trabalho em saúde, além de identificar áreas que necessitam de maior investimento. No Brasil, o Programa Nacional de Melhoria do Acesso e Qualidade na Atenção Básica (PMAQ-AB), que tem suas premissas na Política Nacional da Atenção Básica (PNAB), tem valorizado a avaliação da qualidade da assistência na perspectiva dos usuários, sendo considerada uma das principais contribuições do programa ${ }^{1}$.

Para compreender a atenção básica de forma ampliada, além de valorizar a percepção dos usuários, deve-se reconhecer a influência dos determinantes que podem influenciar o território. No que se refere à saúde infantil, estudos destacam que estas diferenças incidem diretamente na qualidade da assistência e acesso aos serviços².

As Regiões do Brasil com menor Índice de Desenvolvimento Humano (IDH) e cobertura da Estratégia de Saúde da Família (ESF), por exemplo, apresentam maiores dificuldades na oferta de atendimento de qualidade à criança, família e comunidade ${ }^{2,3}$. Portanto estes são indicadores sensíveis a mudanças ligadas aos contextos de vida das famílias.

O Brasil tem apresentado avanços na qualidade da saúde da criança, tais como o incremento dos índices de amamentação exclusiva e redução das taxas de mortalidade infantil até 2017 em 12,8\% ${ }^{4}$. Destaca-se o Programa Nacional de Imunização, considerado o mais exitoso mundialmente e que tem sofrido oscilações regionais nos índices de vacinação, em geral, abaixo do esperado ${ }^{3}$.

A Política Nacional de Atenção Integral à Saúde da Criança tem como eixos principais linhas de cuidado que visam a promoção da saúde, principalmente nos aspectos relacionados ao acompanhamento do crescimento e desenvolvimento infantil, com destaque para os primeiros mil dias de vida. A Estratégia de Saúde da Família, por sua vez, desempenha papel decisivo na articulação e promoção de intervenções para a primeira infância. A capacidade para acessar mulheres e seus filhos durante este período da vida, representa espaço para integrar intervenções eficazes e de baixo custo ${ }^{4}$.

Estudos voltados à análise das ações realizadas pelas equipes de saúde da família (eSF), em geral, avaliam cuidados voltados a um único programa ou assistência em saúde, como por exemplo, foco na amamentação, crescimento e/ou desenvolvimento ${ }^{3,5}$. Tais fatos suscitam estudos que analisem o panorama de determinada região, uma vez que estes são indicadores sensíveis às mudanças ligadas aos contextos das famílias ${ }^{6}$.

Entender estas diferenças é essencial para superar déficits nos indicadores de saúde da criança e a partir deles repensar estratégias de cuidado que alcancem as necessidades da população ${ }^{7}$. Assim, os resultados deste estudo por meio da análise dos dados do terceiro e último ciclo do PMAQ-AB na região Nordeste do Brasil, oportuniza a reflexão da qualidade dos serviços de saúde infantil, mediante a avaliação das usuárias, proporcionando ações mais planejadas que favoreçam o conhecimento na área.

Logo, este estudo traz como contribuição para profissionais, gestores, formuladores de políticas e estudiosos atuantes na área da saúde infantil retratos da heterogeneidade e qualidade da assistência prestada e na produção do conhecimento acerca do alcance da universalidade, integralidade e equidade no âmbito do Sistema Único de Saúde. Desta forma, o objetivo do estudo foi comparar a avaliação das usuárias que participaram do PMAQ-AB acerca da saúde infantil entre os estados da macrorregião nordeste do Brasil.

\section{Métodos}

Trata-se de um estudo transversal, em que foram utilizados dados secundários disponíveis no sítio eletrônico do Ministério da Saúde, mais especificamente no portal do PMAQ. A coleta de dados foi realizada de forma multicêntrica, envolvendo as eSF que atuavam em municípios do Nordeste brasileiro e que aderiram ao $3^{\circ}$ ciclo do PMAQ -AB em 2017-2018.

Neste estudo, foram consideradas elegíveis as mulheres que eram mães/acompanhantes de crianças de 0 a 2 anos de idade, maiores de 18 anos, que realizavam ou realizaram recentemente atendimento de puericultura no serviço e que estavam presentes na UBS no dia da avaliação externa e que não passaram por consulta com médico ou enfermeiro naquele momento. Os critérios de exclusão foram: ser a primeira vez da usuária no serviço ou há mais de 12 meses não ter frequentado a UBS. Os dados coletados foram transferidos em seguida para o banco de dados nacional do Ministério da Saúde (MS).

Para o estudo, foram consideradas 4.190 eSF, correspondendo a $37,28 \%$ do total das eSF avaliadas no país e $99,94 \%$ das equipes da macrorregião Nordeste, compreendida por nove estados a 
saber: Alagoas, Bahia, Ceará, Maranhão, Paraíba, Pernambuco, Piauí, Rio Grande do Norte e Sergipe. Este é o terceiro maior complexo regional do país, ocupando $18,2 \%$ de sua área total.

Os dados são oriundos da etapa de certificação, que contempla a fase da avaliação externa ${ }^{8}$, momento em que um grupo de entrevistadores devidamente capacitados aplicaram instrumentos avaliativos para verificação de padrões de acesso e qualidade da $\mathrm{AB}$ alcançados pelas equipes contratualizadas voluntariamente, sendo conduzida por instituições de ensino e/ou pesquisa contratadas pelo MS. Portanto, o instrumento foi aplicado na própria Unidade Básica de Saúde (UBS) por entrevistador.

A avaliação externa foi desenvolvida em seis módulos distintos ${ }^{8}$. Neste estudo, os dados são oriundos do Módulo III referente à entrevista dos usuários das eSF quanto à disponibilidade e satisfação quanto aos serviços de saúde no que se refere ao seu acesso e utilização ${ }^{9}$. A amostra foi do tipo intencional de no mínimo de 1 e no máximo 4 usuárias por equipe participante do PMA$\mathrm{Q}-\mathrm{AB}$, foi obtida a partir da aceitação de convite para participar da entrevista. Ao todo participaram 5.116 mulheres.

No Módulo III foram utilizadas as questões sobre utilização dos serviços e que compreendiam perguntas sobre a idade da criança. As usuárias foram questionadas com perguntas do tipo sim e não sobre se a consulta até sete dias, se a criança havia sido pesada, medida, colocada para mamar, se teve o umbigo examinado, posição adequada para dormir, recebimento de caderneta da criança, teste do pezinho, se a criança foi atendida continuamente pelos mesmos profissionais, agendamento de consultas, desenvolvimento infantil, alimentação e vacinação, incluindo motivos de não vacinação segundo as respondentes.

$\mathrm{A}$ avaliação externa do $\mathrm{PMAQ}-\mathrm{AB}$ não identificou qualquer usuária que compôs a amostra, preservando-se, assim, a privacidade. A análise descritiva foi utilizada para apresentação dos dados, considerando a frequência absoluta, frequência relativa e intervalo de confiança de 95\% (IC95\%), tanto para a amostral total quanto por estado. As análises foram realizadas utilizando o programa estatístico Stata ${ }^{\circledR}$ v.13.0.

\section{Resultados}

Na Tabela 1 estão apresentadas as frequências absolutas e relativas das características sociodemográficas dos usuários. A maioria dos participan-

Tabela 1. Descrição da amostra de mulheres usuárias e que reportaram ter filhos de até 2 anos de idade da região Nordeste do Brasil. PMAQ-AB 30 ciclo (2017).

\begin{tabular}{|c|c|c|}
\hline Variáveis descritivas & $\mathbf{n}$ & $\%$ \\
\hline \multicolumn{3}{|l|}{ Unidade da Federação (UF) } \\
\hline Alagoas & 330 & 6,3 \\
\hline Bahia & 1211 & 23,1 \\
\hline Ceará & 911 & 17,4 \\
\hline Maranhão & 701 & 13,4 \\
\hline
\end{tabular}

\section{Paraíba}

Pernambuco

Piauí

Sergipe

Estado civil

Solteira

Casada ou união estável

Divorciada/separada

Viúva

Cor de pele/raça declarada

Branca

Preta

Amarela

Parda/mestiça

Indígena

Ignorada

Escolaridade

Não é alfabetizado (não sabe ler e escrever)

É alfabetizado (sabe ler e escrever)

Ensino fundamental incompleto

Ensino fundamental completo

Ensino médio incompleto

Ensino médio completo

Ensino superior incompleto

Ensino superior completo

Pós-Graduação

Não sabe/não respondeu/não lembra

Renda familiar mensal (reais/mês)

\section{até 499}

500-999

1000-1499

1500-1999

2000 ou mais

Faixa-etária (anos)

18-24

25-29

30-34

35-39

$40+$

Fonte:

Ciclo. 
tes eram da Bahia $(23,1 \%)$ e do Ceará $(17,4 \%)$, casadas ou em união estável $(70,6 \%)$, de cor de pele parda ou mestiça segundo autodeclaração (64,8\%), com ensino médio completo $(37,9 \%)$, com salário de 500-999 reais $(50,0 \%)$ e com 18 a 24 anos $(38,9 \%)$.

Na Tabela 2 foram apresentadas as proporções de respostas positivas para os indicadores de saúde da criança para a amostra total e conforme os estados. Quando comparado aos demais estados, o Ceará foi o estado que apresentou melhor cenário com proporções superiores para os seguintes indicadores: consulta até sete dias de vida (73,8\%, IC95\%: 70,8; 76,5); a criança foi pesada $(64,5 \%$, IC95\%: 61,4; 67,6); a criança foi medida (66,1\%, IC95\%: 62,9; 69,1); a criança foi colocada para mamar $(64,3 \%$, IC95\%: $61,2 ; 67,4)$; a criança teve o umbigo examinado (68,5\%, IC95\%: 65,4; 71,4); foi conversado sobre a melhor posição para a criança dormir $(61,3 \%$, IC95\%: 58,0; 64,4); recebimento da caderneta de saúde da criança $(58,8 \%$, IC95\%: 55,6; 62,0); a realização do teste do pezinho até os sete dias de vida (92,1\%, IC95\%: 90,2; 93,7).

Em todos os estados a prevalência de crianças com todas as vacinas foi acima de $90 \%$, sem diferenças significativas. Ao avaliar se as equipes das unidades básicas de saúde já procuraram devido ao atraso nas vacinas das crianças, todos os estados apresentaram baixas prevalências. Verificouse que, em quase todos os estados, a maioria das crianças já sai com a próxima consulta marcada, com exceção dos estados de Alagoas (48,2\%, IC95\%: 42,8; 53,6), Maranhão (35,1\%, IC95\%: $31,6 ; 38,7)$ e Sergipe $(36,8 \%$, IC95\%: 30,7; 43,3). Maiores detalhes dos indicadores de saúde podem ser observados na Tabela 2.

\section{Discussão}

A distribuição dos indicadores de saúde da criança entre os estados da macrorregião Nordeste mostrou avanços nas ações voltadas à saúde da criança, como a realização do teste do pezinho até sete dias de vida, criança sempre consultada pelos mesmos profissionais, acompanhamento do crescimento e desenvolvimento esperado para a idade, orientação alimentar e vacinação em dia. Por outro lado, cuidados relativos a colocar a criança para mamar nos primeiros dias de vida e informação aos pais sobre a melhor forma da criança dormir, foram ações pouco relatadas pelas usuárias.
Dentre os estados, o Ceará apresentou melhores resultados na qualidade da atenção à saúde da criança. Esta informação reflete o compromisso dos estados no cuidado neonatal, período ideal para orientações e apoio ao aleitamento materno exclusivo, imunizações, acompanhamento do crescimento/desenvolvimento e realização das triagens neonatais ${ }^{10}$. Além disso, tanto a Rede Cegonha quanto a Política Nacional de Atenção Integral à Saúde da Criança determinam ações e cuidados nesse período, com destaque para o " 5 " dia de saúde integral", que incentiva a puérpera e o recém-nascido a irem à Unidade de Saúde para realizar os cuidados necessários, como a checagem das vacinas contra Hepatite B e a BCG e realizarem a consulta de puericultura ${ }^{11}$.

No estudo verificou-se que a avaliação das medidas antropométricas e observação do coto umbilical do recém-nascido, atividades essenciais durante a avaliação da criança, são realizadas em média, apenas por metade das equipes, segundo as usuárias. O Ceará apresentou a maior média com $66,1 \%$, enquanto a Paraíba 33,4\%, evidenciando diferenças importantes entre as regiões.

Estudo que comparou a atenção à saúde da criança em dois municípios da Paraíba, com classificação de pequeno e médio porte, identificou que as crianças eram mais medidas do que pesadas, mesmo com a presença de balança infantil e antropômetro nas $\mathrm{UBS}^{12}$. Um estudo com dados do $1^{\circ}$ ciclo do PMAQ-AB nas capitais brasileiras, identificou-se adequação da estrutura de UBS para o atendimento infantil em apenas em 35,8\% das $\mathrm{UBS}^{13}$.

Internacionalmente esta realidade é observada em estudo produzido no México onde as intervenções em saúde materno-infantil em municípios de menor porte possuem barreiras na qualidade da assistência principalmente quando distantes dos grandes centros urbanos ${ }^{14}$. Esta análise evidencia a importância da equidade na gestão pública de recursos e demonstra as dificuldades dos municípios menores em prestar a longitudinalidade do cuidado, principalmente ao neonato precoce na atenção básica ${ }^{15}$.

Em relação a falar sobre a melhor posição para dormir a fim de evitar a síndrome da morte súbita do lactente (SMSL), apenas $45,7 \%$ das usuárias relataram esta orientação. O Ceará orientou as famílias em cerca de $61,3 \%$ obtendo o maior percentual. A SMSL é uma das principais causas de morte na infância e a faixa etária de maior risco se encontra entre dois e cinco meses de vida, e ocorre principalmente em famílias em situação de vulnerabilidade social e econômica. 


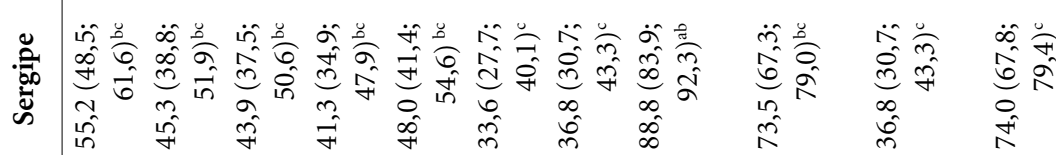

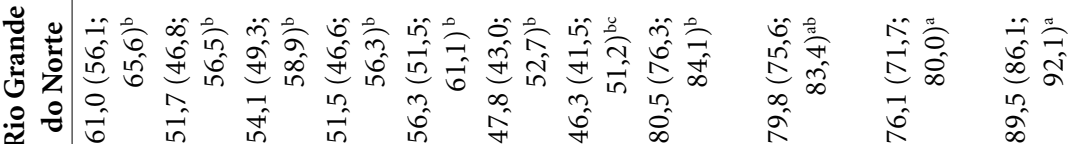

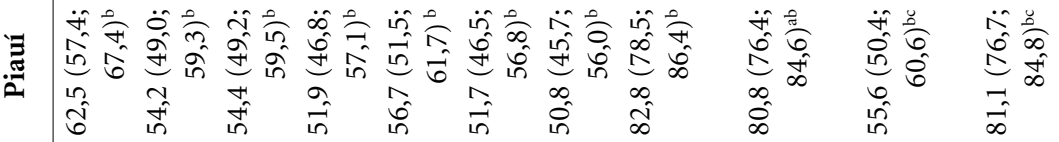

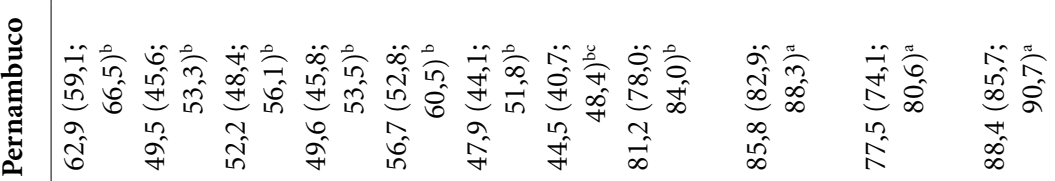

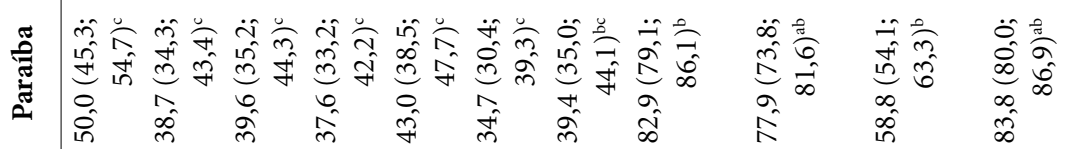

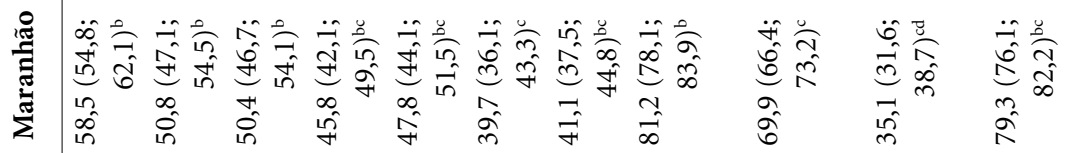

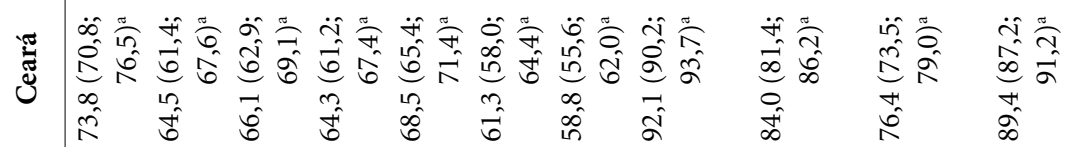

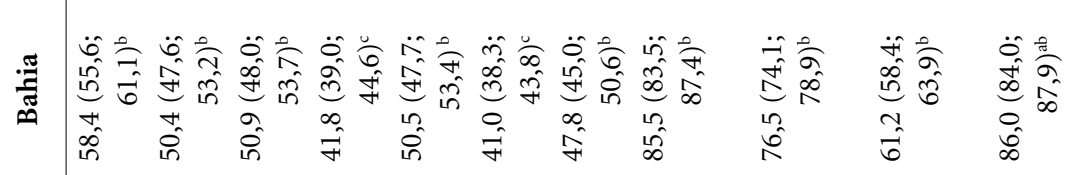

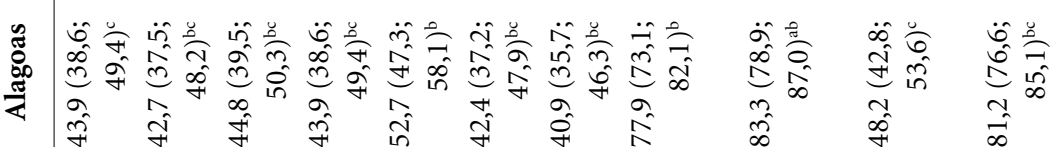

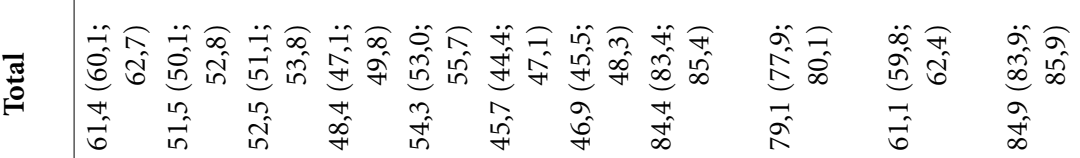

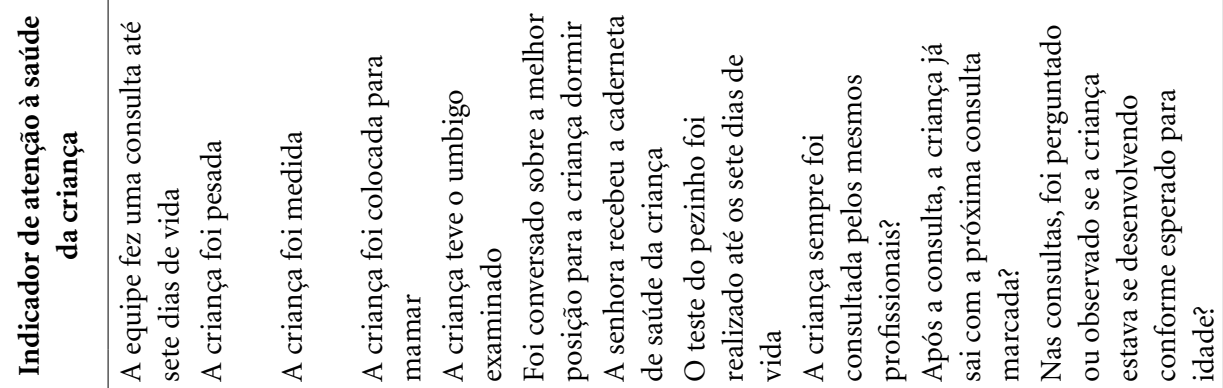


Tal resultado sugere que os profissionais de saúde devem abordar o sono infantil durante as consultas de puericultura, com destaque para a fase de lactância ${ }^{16}$ e não somente as medidas antropométricas e ao preenchimento de gráficos, uma vez que o caráter educativo da consulta e a comunicação com a família é fator essencial para a promoção da saúde.

Sobre o recebimento da caderneta de saúde da criança, a maioria das usuárias respondeu que não. No estado do Ceará, com a maior média para este indicador, apenas $58,8 \%$ respondeu que sim, ainda aquém do recomendado pela Rede Cegonha. Este achado vai de encontro à literatura nacional que aponta uma variação entre 33\% e $59,6 \%$ no recebimento da caderneta e/ou informações sobre como utilizá-la ${ }^{17}$. Estes resultados apontam que a falta desse insumo dificulta o acompanhamento infantil e pode desmotivar os pais pelo não reconhecimento da importância da puericultura e acompanhamento da criança na $\mathrm{AB}$, além disso mesmo com o uso do prontuário eletrônico, por exemplo, registrar as informações na caderneta facilita inclusive a coordenação do cuidado da criança.

Perguntar sobre a realização do teste do pezinho com até sete dias de vida, obteve um percentual de respostas positivas acima de 70\% em todos os estados, corroborando com uma pesquisa realizada com 64 mães sobre o teste do pezinho, no qual $55(85,9 \%)$ sabiam que deveria ser feito no período de até sete dias, viabilizando assim a execução do teste ${ }^{18}$.

Quanto à longitudinalidade do cuidado, a grande maioria das equipes $(79,1 \%)$ segundo as usuárias, mantém o vínculo, onde o mesmo profissional acompanha a criança ao longo do tempo, refletindo uma relação interpessoal que expressa a confiança mútua entre as famílias e profissionais de saúde. Estudos apresentam evidências consistentes para a associação entre maior grau de orientação à APS e o aumento da efetividade dos sistemas de saúde ${ }^{19}$.

No entanto, o relato sobre o agendamento das consultas subsequentes foi considerado uma fragilidade, com destaques para os estados do Maranhão (35,1\%) e Sergipe (36,8\%). O agendamento prévio está atrelado ao atributo de primeiro contato, princípio essencial da atenção primária, o que aponta a fragilidade na organização do serviço para atendimento do usuário em tempo oportuno. Essas barreiras são mais nítidas nas regiões de saúde de baixa oferta e desenvolvimento socioeconômico ${ }^{20}$. Baixos níveis de longitudinalidade e coordenação do cuidado devem ser discutidos pelas eSF e podem ser superados com apoio do agente comunitário de saúde, uso de prontuário eletrônico integrado, participação em telessaúde, apoio de especialistas da rede, residências em saúde e fluxos institucionais de comunicação, entre outros ${ }^{21}$ incluindo a participação dos profissionais em mestrados profissionais na área.

Acerca da observação dos profissionais sobre o desenvolvimento infantil observa-se pouca variação entre os estados, variando de 74\% (Sergipe) a $89,5 \%$ (Rio Grande do Norte). Esse fato merece destaque visto que estas ações realizadas nas unidades de saúde devem ser de interesse de todos os componentes da eSF. Portanto os integrantes da equipe devem analisar de forma crítica, reconhecendo os marcos do desenvolvimento infantil e detectando precocemente possíveis atrasos. A família também precisa ser envolvida nesse momento, para que possa realizar escolhas que promovam a saúde infantil e que seja capaz de reconhecer sinais de alerta. Essa atenção é uma forma de prevenção e promoção da saúde, contribuindo para um crescimento e desenvolvimento plenos ${ }^{22}$.

No processo de desenvolvimento infantil, os hábitos alimentares das crianças até os dois anos, são grandes aliados, cuja prática de orientação foi superior a $76 \%$ em todos os estados. O Ministério da Saúde enfatiza que em todas as etapas da vida torna-se fundamental a orientação de hábitos alimentares, merecendo destaque os primeiros anos, que são fundamentais para o crescimento e desenvolvimento satisfatório, bem como para a manutenção da saúde ${ }^{23}$. Em contrapartida, estudo realizado com 709 mães detectou-se que menos da metade $(48,4 \%)$ foram orientadas sobre a introdução da alimentação complementar (AC) e quando analisado esse conhecimento, os resultados apontam falhas, no que concerne a introdução da $\mathrm{AC}$, no qual sugeriram que as ações educativas em relação à alimentação infantil podem não estar sendo realizadas a contento e necessitam ser intensificadas, principalmente no que se refere à introdução da $\mathrm{AC}^{24}$.

Um ponto positivo foi o percentual elevado de usuárias que indicaram que as crianças foram vacinadas, com destaque para o estado do $\mathrm{Ma}$ ranhão, onde $97,1 \%$ das crianças estavam com o cartão de vacina atualizado. Estes dados podem estar relacionados ao fato de que o abastecimento das vacinas e a logística tem forte influência do Programa Nacional de Imunização (PNI), cabendo às unidades de saúde adequação conforme as normas técnicas do Ministério da Saúde ${ }^{25}$. 
Estudo realizado no Ceará que analisou dados do PMAQ reforçou que os itens avaliados relativos à vacinação também foram bem avaliados nos dois ciclos anteriores do PMAQ, pois os municípios têm baixa interferência nesta atividade, já que têm maior dependência do $\mathrm{MS}^{26}$.

Ainda sobre a vacinação, as mães que não estavam com o cartão atualizado referiram entre as barreiras, a falta de vacina na unidade. Ressalta-se que esse achado corresponde a uma dificuldade na utilização de serviços de saúde e a oportunidades perdidas de vacinação, o que pode comprometer o cumprimento das coberturas vacinais. Estudo ${ }^{27}$ apontou que a falta destes insumos na unidade de saúde, constitui um obstáculo para a cobertura vacinal adequada.

A partir do exposto, verifica-se que a maioria das ações de saúde da criança avaliados pelo PMAQ são de baixa complexidade e muitas vezes não necessitam de tecnologia dura, mas poderiam ser realizadas na medida em que valorizassem o diálogo aberto entre profissional e a mãe/ cuidador, a fim de esclarecer sobre a influência destes indicadores na saúde infantil ${ }^{28}$.

As avaliações dos serviços de assistência à criança buscam otimizar os processos e reduzir a incidência de doenças, apresentando relação direta com a vinculação ao serviço e aos profissionais de saúde. Entretanto, a acessibilidade ainda é apontada como forte limitante da qualidade da APS, principalmente entre as populações mais vulneráveis e localidades com menos recursos e com mais dificuldade para fixação de profissionais ${ }^{29}$.

Estes resultados poderão contribuir para a superação dos desafios que limitam o pleno funcionamento da APS, sensibilizando gestores sobre a importância desta área estratégica, reorganizando a atenção à saúde e as linhas de cuidado para esta clientela através das tecnologias de cuidado, e contribuindo para o desenvolvimento infantil ${ }^{30}$.

\section{Limitações}

O PMAQ é um programa em que as eSF não tem obrigatoriedade de adesão, mas que para sua mobilização, o Ministério da Saúde utilizou a estratégia de indução financeira com repasse de recursos às equipes, articulando-as ao desempe- nho da eSF a partir dos resultados do programa, conforme padrões específicos que poderiam ser na ampliação do acesso aos serviços, ou melhoria nas condições de trabalho e/ou no desenvolvimento dos profissionais da saúde. Cada município teve autonomia para decidir as aplicações de seus recursos. Tal situação pode ter superestimado alguns resultados influenciando a avaliação devido ao interesse direto dos participantes. Além disso, no que se refere as usuárias, foi utilizada amostra por conveniência, portanto suas conclusões são vulneráveis ao viés de seleção. Soma-se o fato de que ao longo do tempo o instrumento sofreu variações, tornando inviável comparações ao longo dos ciclos o que pode ser uma limitação do estudo ou discussão com a literatura pertinente. Outro ponto a ser discutido é que muitas vezes os municípios menores com baixa qualidade na atenção à saúde da criança, podem influenciar a média das atividades por Estado, o que de fato não reflete o contexto dos municípios em geral. Para isso, recomenda-se que análises dentro do contexto municipal possam ser realizadas.

\section{Conclusão}

Embora os estados do Nordeste brasileiro tenham avançado na atenção ao cuidado da criança, fortalecendo as políticas de cuidado na ESF, os indicadores analisados apontam desigualdades na macrorregião de estudo. Os fatores econômicos, sociais e demográficos permanecem influenciando na qualidade da assistência ofertada. Isso reforça a necessidade constante de avaliações do processo de trabalho, a divulgação dos dados e reformulações dos objetivos da eSF, em conjunto com gestores e comunidade.

Neste sentido, a saúde infantil deve ser uma das prioridades de atenção, principalmente para as crianças em maior situação de vulnerabilidade, como as prematuras e de baixo peso, no intuito de evitar agravos e mortes evitáveis por meio de ações de cuidados básicos. Esse acompanhamento mais próximo deve ser associado às atividades em domicílio e na comunidade, os quais permitem uma visão mais holística dos fatores que influenciam o crescimento e desenvolvimento da criança. 


\section{Colaboradores}

FA Gubert: concepção e desenho ou análise e interpretação dos dados; redigir o artigo ou revisá-lo criticamente; e aprovação da versão a ser publicada. VC Barbosa Filho: a concepção e desenho ou análise e interpretação dos dados; redigir o artigo ou revisá-lo criticamente; e aprovação da versão a ser publicada. RCS Queiroz: concepção e desenho ou análise e interpretação dos dados; redigir o artigo ou revisá-lo criticamente; e aprovação da versão a ser publicada. MC Martins: concepção e desenho ou análise e interpretação dos dados; redigir o artigo ou revisá-lo criticamente; e aprovação da versão a ser publicada. RS Alves: concepção e desenho ou análise e interpretação dos dados; redigir o artigo ou revisá-lo criticamente; e aprovação da versão a ser publicada. ILTP Rolim: concepção e desenho ou análise e interpretação dos dados; redigir o artigo ou revisá-lo criticamente; e aprovação da versão a ser publicada. MSV Lopes: concepção e desenho ou análise e interpretação dos dados; redigir o artigo ou revisá-lo criticamente; e aprovação da versão a ser publicada. APGF Vieira-Meyer: concepção e desenho ou análise e interpretação dos dados; revisão de redação e edição - igual.

\section{Agradecimentos}

A Rede Nordeste de Formação em Saúde da Família (RENASF) e a Fundação de Amparo à Pesquisa e ao Desenvolvimento Científico e Tecnológico do Maranhão (FAPEMA).

\section{Referências}

1. Figueiredo DCMM, Shimizu HE, Ramalho WM, Figueiredo AM, Lucena KDT. Quality of primary health care in Brazil: patients' view. Rev Bras Enferm 2018; 71(Supl. 6):2713-2719.

2. Santos AS, Duro SMS, Cade NV, Facchini LA, Tomasi E. Quality of infant care in primary health services in Southern and Northeastern Brazil. Rev Saude Publica 2018; 52:11.

3. Bousquat A, Giovanella L, Fausto MCR, Fusaro ER, Mendonça MHM, Gagno J, Viana ALA. Tipologia da estrutura das unidades básicas de saúde brasileiras: os 5 R. Cad Saude Publica 2017; 33(8):e00037316.

4. Leal MC, Szwarcwald CL, Almeida PVB, Aquino EML, Barreto ML, Barros F, Victora C. Reproductive, maternal, neonatal and child health in the 30 years since the creation of the Unified Health System (SUS). Cien Saude Colet 2018; 23(6):1915-1928.

5. Jaime PC, Frias PG, Monteiro HOC, Almeida PVB, Malta DC. Healthcare and unhealthy eating among children aged under two years: data from the National Health Survey, Brazil, 2013. Rev Bras Saude Mater Infant 2016; 16(2):149-157.

6. Feitosa ANC, Oliveira CL, Duarte EB, Oliveira AM. Avaliação em saúde: uma revisão integrativa. Id on Line Rev Mult Psic 2016; 10(30):274-281.

7. Pinto CAG, Paraguay NLBB, Pereira JO. Estudos dos indicadores epidemiológicos de saúde da criança na Rede Cegonha. Divulg Saúde Debate 2016; 55:172-216.

8. Brasil. Ministério da Saúde (MS). Programa Nacional de Melhoria do Acesso e da Qualidade da Atenção Básica (PMAQ): Manual Instrutivo $3^{\circ}$ Ciclo (2015 - 2016). Brasília: MS; 2015.

9. Brasil. Ministério da Saúde (MS). Manual instrutivo do Pmaq para as equipes de atenção básica (saúde da família, saúde bucal e equipes parametrizadas) e Nasf. 2a ed. Brasília: MS; 2015.

10. Furtado MCC, Mello DF, Pina JC, Vicente JB, Lima PR, Rezende VD. Nurses' actions and articulations in child care in primary health care. Texto Contexto Enferm 2018; 27(1):e0930016.

11. Brasil. Ministério da Saúde (MS). Portaria no 1.130 , de 5 de agosto de 2015. Institui a Política nacional de Atenção Integral à Saúde da Criança, PNAISC. Diário Oficial da União 2015; 6 ago.

12. Pedraza DF, Santos IS. Assessment of growth monitoring in child care visits at the Family Health Strategy in two of Paraíba State, Brazil. Epidemiol Serv Saúde 2017; 26(4):847-855.

13. Lisboa LAS, Queiroz RCS, Thomaz EBAF, Silva NC, Rocha TAH, Vissoci JRN, Staton CA, Lein A, Simões VMF, Thumé E, Facchini LA. Characteristics of primary care and rates of pediatric hospitalizations in Brazil. Rev Saude Publica 2020; 54:32.

14. Ramírez-Tirado LA, Tirado-Gómez LL, Lópes-Cervantes M. Desigualdad en intervenciones de atención primaria para el cuidado de la salud materno infantil en México. Rev Panam Salud Pública 2014; 35(4):235241.

15. Lima JG, Giovanella L, Fausto MCR, Bousquat A, Silva EV. Atributos essenciais da atenção primária à saúde: resultados nacionais do PMAQ-AB. Saúde Debate 2018; 42(esp. 1):52-66. 
16. Oliveira AMF, Andrade PR, Pinheiro EM, Avelar AFM, Costa P, Belela-Anacleto ASC. Risk and protective factors for sudden infant death syndrome. Rev Bras Enferm 2020; 73(2):e20190458.

17. Sousa JCB, Silva RD, Olivindo DDF. Os registros da caderneta de saúde da criança no acompanhamento do crescimento e desenvolvimento infantil. Res Soc Develop 2020; 9(10):e6209109017.

18. Arduini GAO, Balarin MAS, Silva-Grecco RL, Marqui ABT. Conhecimento das puérperas sobre o teste do pezinho. Rev Paul Pediatr 2017; 35(2):151-157.

19. Harzheim E, Pinto L, Hauser L, Soranz D. Avaliação dos usuários crianças e adultos quanto ao grau de orientação para Atenção Primária à Saúde na cidade do Rio de Janeiro, Brasil. Cien Saude Colet 2016; 21(5):1399-1408.

20. Lima JG, Giovanella L, Fausto MCR, Bousquat A. Qualidade da atenção básica por tipos de regiões de saúde. Novos Caminhos 2016; 12:1-76.

21. Cruz MJB, Santos AF, Araújo LHL, Andrade EIG. A coordenação do cuidado na qualidade da assistência à saúde da mulher e da criança no PMAQ. Cad Saude Publica 2019; 35(11): e00004019.

22. Almeida AC, Costa ML, Sad IR, Ramos EG, Fonseca VM, Peixoto MVM. Use of a monitoring tool for growth and development in Brazilian children: systematic review. Rev Paul Pediatr 2016; 34(1):122-131.

23. Brasil. Ministério da Saúde (MS). Guia alimentar para crianças brasileiras menores de 2 anos. Brasília: MS; 2019.

24. Pizzatto P, Dalabona CC, Correa ML, Neumann NA, Cesar JA. Maternal knowledge on infant feeding in São Luís, Maranhão, Brazil. Rev Bras Saude Mater Infant 2020; 20(1):169-179.

25. Siqueira LG, Martins AMEBL, Versiani CMC, Almeida LAV, Oliveira CS, Nascimento JE, Alecrim BPA, Bezerra RC. Avaliação da organização e funcionamento das salas de vacina na Atenção Primária à Saúde em Montes Claros, Minas Gerais, 2015. Epidemiol Serv Saude 2017; 26(3):557-568.
26. Vieira-Meyer APGF, Morais APP, Guimarães JMX, Campelo ILB, Vieira NFC, Machado MFAS, Nogueira PSF, Nuto SAS, Freitas RWJF. Infrastructure and work process in primary health care: PMAQ in Ceará. Rev Saude Publica 2020; 54:62.

27. Ferreira AV, Oliveira CF, Guimarães EAA, Cavalcante RB, Moraes JT, Oliveira VC. Acesso à sala de vacinas nos serviços de atenção primária à saúde. Rev Eletr Enf 2017; 19:1-11.

28. Damaceno NA, Bandeira D, Hodali N, Weiller TH. Access at first contact in primary health care: integrative review. Rev APS 2016; 19(1):122-138.

29. Silva GS, Fernandes DRF, Alves CRL. Avaliação da assistência à saúde da criança na Atenção Primária no Brasil: revisão sistemática de métodos e resultados. Cien Saude Colet 2020; 25(8):3185-3200.

30. Ferreira, TLS, Costa ICC, Andrade FB. Avaliação do atributo integralidade em serviços de puericultura na atenção primária à saúde. Rev Cien Plural 2015; $1(1): 22-29$.

Artigo apresentado em 06/08/2020

Aprovado em 04/03/2021

Versão final apresentada em 06/03/2021

Editores-chefes: Romeu Gomes, Antônio Augusto Moura da Silva 